\title{
Estrogen is a novel regulator of Tnfaip1 in mouse hippocampus
}

\author{
HUI LIU ${ }^{1,2^{*}}$, LIPING YANG ${ }^{2,4^{*}}$, YINGCHUN ZHAO ${ }^{3 *}$, GUIHUA ZENG $^{2}$, YAOSONG WU $^{5}$, \\ ${\text { YULONG } \mathrm{CHEN}^{5} \text {, JIAN ZHANG }}^{4}$ and QINGRU ZENG ${ }^{1}$
}

\begin{abstract}
${ }^{1}$ Department of Life Science, Hunan Agricultural University, Changsha, Hunan 410128; ${ }^{2}$ Department of Enviromental Science, Changsha Environmental Protection College, Changsha, Hunan 410004, P.R. China; ${ }^{3}$ Department of Biostatistics and Bioinformatics, Tulane University, New Orleans, LA 70112, USA; ${ }^{4}$ Key Laboratory of Protein Chemistry and Developmental Biology, Ministry of Education of China, Department of Biochemistry and Molecular Biology, College of Life Science, Hunan Normal University, Changsha, Hunan $410081 ;{ }^{5}$ Laboratory of Molecular Biology, Henan University of Traditional Chinese Medicine, Zhengzhou, Henan 410208, P.R. China
\end{abstract}

Received November 2, 2013; Accepted February 26, 2014

DOI: $10.3892 / \mathrm{ijmm} .2014 .1742$

\begin{abstract}
Tumor necrosis factor-induced protein 1 (Tnfaip1), also known as B12, has been previously identified as a tumor necrosis factor- $\alpha$ (TNF- $\alpha)$-inducible protein and is involved in the cytokinesis signaling pathway, DNA synthesis, innate immunity, cell apoptosis, Alzheimer's disease (AD) and type 2 diabetic nephropathy. However, little is known regarding the expression of Tnfaipl in various tissues or its accurate role in these physiological functions. The focus of this study was on Tnfaipl expression in different tissues, with a high expression in mouse hippocampus being identified. The age- and gender-related expression of Tnfaip1 in hippocampus was also investigated. The distribution of Tnfaipl was mapped using fluorescent immunostaining. Although immunoactivity was found in the CA1, CA3 and DG subregions of the hippocampus in E17.5 and P6 mice, strong staining was only detected in the CA3 subregion in adult mice. These data suggested that Tnfaip1 expression in hippocampus may be regulated by estrogen. Further study showed that the expression of Tnfaip1 in the hippocampus was significantly increased in ovariectomized mice compared to Sham mice. In cultured primary hippocampal cells, Tnfaip1 showed different expression levels
\end{abstract}

Correspondence to: Professor Qingru Zeng, Department of Life Science, Hunan Agricultural University, Agricultural Road No. 1, Changsha, Hunan 410128, P.R. China

E-mail: liuhui@hunnu.edu.cn

Professor Jian Zhang, Key Laboratory of Protein Chemistry and Developmental Biology, Ministry of Education of China, Department of Biochemistry and Molecular Biology, College of Life Science, Hunan Normal University, 36 Lushan Road, Changsha, Hunan 410081, P.R. China

E-mail: zhangjian@hunnu.edu.cn

${ }^{*}$ Contributed equally

Key words: estrogen, tumor necrosis factor induced protein 1, regulator, hippocampus in different treatments of estrogen or estrogen receptor antagonists. Additional experiments demonstrated the existence of a binding site of ER $\beta$ in the Tnfaip1 promoter region, and that ER $\beta$ was able to upregulate Tnfaip1 expression. Our study identified a new regulatory factor and a primary regulatory mechanism of Tnfaipl expression in hippocampus. Since both hippocampus and estrogen are crucial in $\mathrm{AD}$, the results also showed a potential association between Tnfaipl and hippocampal-related diseases, such as AD, which may be affected by the estrogen level.

\section{Introduction}

Tumor necrosis factor-induced protein 1 (Tnfaip1), which was also termed B12 and previously identified as a tumor necrosis factor- $\alpha$ (TNF- $\alpha$ )-inducible protein (1), is highly conserved in a wide range of species including human (1), mouse (1), rat (2), and Caenorhabditis elegans (C.elegans) (3). In mammals, Tnfaip1 has at least two paralogs, KCTD10 and KCTD13, which share $>60 \%$ amino acid sequence identities, and a conserved BTB/POZ domain at their N-terminus. Besides sequence similarity, all of the paralogs show some common functional features. They may play roles in the cytokinesis signaling pathway and DNA synthesis by interacting with proliferating cell nuclear antigen (PCNA) and small subunit (p50) of polymerase $\delta(2,4,5)$. Tnfaipl is also involved in the process of Alzheimer's disease (AD) development (3), the innate immunity against hepatitis B virus (HBV) (6), the apoptosis of cancer cells (7), the phosphorylation procedure (8) and type 2 diabetic nephropathy (9). Tnfaip1 is differentially expressed in various tissues such as liver, kidney, heart, placenta and brain of developing mouse embryo (1) and has a lower expression in cancer cells (7). However, the detailed expression of Tnfaip1 and its exact function in these physiological processes has yet to be thoroughly investigated. The detailed expression in nerve system has also not been reported, particularly in hippocampus, which is one of the brain regions affected early in AD, although it was previously reported that Tnfaipl was involved in response to $\beta$-amyloid peptide accumulation in a transgenic C. elegans AD model (3). 
The sex hormone estrogen has multiple functions, including the differentiation and function of the reproductive tract, memory storage and bone growth $(10,11)$. Usually, estrogen exhibits its function by binding to the classic estrogen receptors, ER $\alpha$ and ER $\beta$, which are nuclear transcription factors that activate or repress gene expression (12). Hippocampal neurons express ER $\alpha$ and ER $\beta$ mRNA in tissue sections as well as in slice cultures and dispersion cultures $(13,14)$. Results of previous studies strongly suggest that hippocampal neurons are able to synthesize estrogens de novo $(14,15)$, and hippocampus-derived endogenous estrogen is essential for hippocampal synaptic plasticity (16). Peripheral estrogen has also been found to exert a protective effect in hippocampal neurons (17). For more than two decades, it is known that estrogens influence synaptic plasticity in the hippocampus. Gould et al (18) showed that ovariectomy (OVX) of female rats resulted in a decrease of dendritic spine density on CA1 pyramidal neurons in the hippocampus. Accordingly, systemic estradiol treatment of these ovariectomized animals caused an increase in the number of dendritic spines in this particular hippocampal region. Consistently, spine synapse density varied in response to fluctuating estrogen levels during the estrous cycle in female rats (19).

In this study, in order to study the function of Tnfaip1 in physiological processes, such as AD disease, we focused on the Tnfaipl expression in mouse hippocampus. Results of the present study showed that Tnfaipl expression is associated with age and gender, possibly regulated by estrogen in hippocampus. This result is supported by in vitro and in vivo studies. Through promoter analysis, a potential binding site for ER $\beta$ was identified in the Tnfaipl promoter region and Tnfaipl expression was upregulated by ER $\beta$. The identification of a novel regulatory factor and mechanism for Tnfaip1 in hippocampus indicates the involvement of Tnfaip1 in hippocampus-related disease, such as AD.

\section{Materials and methods}

Mice and estrogen manipulations. C57Bl/6J (B6) mice were housed in a 12:12 h light:dark cycle. Sham, OVX and $17 \beta$-estradiol pellet implantation were performed as previously described $(20,21)$. All the OVX $(n=12)$ and Sham $(n=6)$ mice were 2 months old. The OVX mice had access to phytoestrogen-free food, receiving either a placebo pellet $(\mathrm{OVX}+$ placebo; $\mathrm{n}=6)$ or a $17 \beta$-estradiol pellet $(\mathrm{OVX}+\mathrm{E} 2$; $\mathrm{n}=6)$. The pellets contained $0.18 \mathrm{mg}$ of $17 \beta$-estradiol or placebo in a matrix that is designed to release contents (17 $\beta$-estradiol or placebo) over a 60-day period (Innovative Research of America, Sarasota, FL, USA). Sham operations were performed (Sham; $n=6$ ) on an additional group of mice. Treatment with placebo or $17 \beta$-estradiol lasted for 4 weeks. Unless otherwise indicated, the reported results were obtained from homozygous mutants. The animal procedures were approved by the Institutional Animal Care and Use Committee of Hunan Agriculture University (approval no. 09-01).

Primary hippocampal neuronal culture. Hippocampal neuronal cells were prepared from mouse embryos using the methods described previously (22). Briefly, female C57Bl/6J (B6) mice with 18.5 days of gestation were anesthetized with isoflurane, and embryonic mouse pups were surgically removed and decapitated. Hippocampuses were harvested under sterile conditions. The hippocampal tissue was enzymatically dissociated in $0.125 \%$ trypsin II (Sigma-Aldrich, St. Louis, MO, USA). Isolated neural cells were placed in poly-D-lysine-coated 35-mm plastic culture dishes containing $3 \mathrm{ml}$ of neurobasal medium to a culture surface cell density of $5 \times 10^{5} / \mathrm{ml}\left(400-500 / \mathrm{mm}^{2}\right)$. The cultures were maintained in neurobasal medium supplemented with B27 (2\%, v/v; Invitrogen, Carlsbad, CA, USA), glutamine $(0.5 \mathrm{mM})$ and penicillin/streptomycin (100 units) for at least 7-10 days prior to being used for experiments. To test the potential function of ERs, hippocampal neuronal cultures were treated with $1 \mu \mathrm{M}$ ER antagonist ICI182,780 (Sigma-Aldrich), or $1 \mu \mathrm{M}$ selective ER $\alpha$ antagonist methyl-piperidino-pyrazole (MPP; Sigma-Aldrich), or $5 \mu \mathrm{M}$ selective ER $\beta$ antagonist 4-[2-phenyl5,7-bis(trifluoromethyl)pyrazolo[1,5-a]pyrimidin-3-yl] phenol (PHTPP; Sigma-Aldrich), with the presence of $10 \mathrm{nM}$ $17 \beta$-estradiol (E2; Sigma-Aldrich). The antagonists and E2 were dissolved in dimethylsulfoxide (DMSO). Vehicle-treated controls received the same volume of DMSO. Medium was changed with fresh medium every 4 days.

Quantitative real-time-polymerase chain reaction ( $q R T-P C R$ ). Total RNA was prepared using TRIzol (Invitrogen), and an equal amount of RNA from each tissue (50-100 ng) was reverse transcribed using the SuperScript III first-strand synthesis system (Invitrogen). SYBR-Green PCR Master Mix for qRT-PCR was purchased from ABI (Applied Biosystems, Foster City, CA, USA). qRT-PCR was performed according to the manufacturer's instructions, with minor modifications based on cDNA samples and primers. The primers used in this study were: Tnfaipl forward, 5'-gcgtgctcttcatcaaggat-3' and reverse, 5'-ttccaccttggtctgcttct-3'; $\beta$-actin forward, 5'-cggttccgatgecc tgaggctctt-3' and reverse, 5'-cgtcacacttcatgatggaattga-3'; and 40 cycles of amplification was achieved in a 96-well plate consisting of SYBR-Green Master Mix, primers and cDNA. The standard mode of an ABI 7500 Fast Sequence detection system was used. $\beta$-actin was amplified in parallel as an endogenous control to standardize the amount of sample and for the quantification of the relative gene expression within and between samples. Reactions were performed in triplicate.

Fluorescent immunostaining. Frozen tissue sections (10 mm) were treated with blocking solution containing $5 \%$ goat serum and $0.2 \%$ Triton X-100 at room temperature for $30 \mathrm{~min}$. Primary antibodies were added at a dilution of 1:200, and incubated at $4^{\circ} \mathrm{C}$ overnight. After three washes in $1 \mathrm{X}$ phosphate-buffered saline (PBS), Alexa-568 (Molecular Probes, Carlsbad, CA, USA) conjugated secondary antibodies were added at a dilution of 1:600, together with DAPI $(1: 10,000)$, and incubated at room temperature for $2 \mathrm{~h}$ in the dark. Non-immune sera (instead of primary antibodies) were used as negative controls in the preliminary experiment. Slides were mounted in Fluoromount-G (Southern Biotech, Birmingham, AL, USA) and viewed using a Carl Zeiss Microscope Objective (Microscope Axio Observer. A1; Carl Zeiss Microscopy, LLC, San Diego, CA, USA).

Western blot analysis. To study the effect of estrogen on the expression of Tnfiapl in vitro and in vivo, western blot assay was performed as previously described (8). The proteins were extracted with RIPA buffer and boiled together with 
loading buffer. After separation with SDS/PAGE, the proteins were transferred to a polyvinylidene difluoride membrane (Millipore), blocked in 5\% non-fat milk for $1 \mathrm{~h}$, and probed overnight with the rabbit polyclonal anti-Tnfaip1 (Abcam) in the presence of $5 \%$ non-fat milk. Immunoblots were washed 3 times (5-10 min each) in PBS containing 0.1\% Tween-20 and incubated with the goat anti-rat-HRP (Amersham Biosciences) $(1: 10,000)$ for $1 \mathrm{~h}$. Blots were washed 3 times $(10 \mathrm{~min}$ each $)$ in PBS containing $0.1 \%$ Tween-20, developed in ECL reagent (Millipore) for 2-5 min, and exposed to Blue XB-1 film (Kodak).

Promoter analysis. The promoter region of mouse Tnfaip1 (GenBank accession no. NM_009395.4) was predicted using the Promoter Inspector program (www.genomatix.de) and ProScan (www.bimas.cit.nih.gov). Transcription factor binding sites were predicted by online software JASPAR (http://jaspar. cgb.ki.se/). The CpG were also predicted by online software (http://www.bio-soft.net/sms/cpg_island.html). The promoter region (-2166/+61) was amplified from mouse genomic DNA using primer pairs with $K p n I$ site at the forward primers and XhoI site at the reverse primers. A series of fragments including $(-1072 /+61),(-734 /+61)$ and $(-273 /+61)$ were amplified from the promoter region. The primers used were: forward primers with KpnI site, for -2166/+61, 5'-ggggtacc cagacacgtgaacctgaagga-3'; -1072/+61, 5'-ggggtacctgataaccgctagcagtggat-3'; -734/+61, 5'-ggggtaccgatgggtaagatagatatggt-3'; -273/+61, 5'-ggggtaccg tcccaagtcctccacg-3'; the common reverse primer with XhoI site, 5'-ccgctcgagagcagtgggtcgaaaacttgac-3'. The letters in bold italic refer to the recognition site of the restriction enzyme. These amplified fragments were inserted into the promoterlessluciferase gene upstream of plasmid pTAL-luc, separately denoted p(-2166/+61)-Luc, p(-1072/+61)-Luc, p(-734/+61)-Luc and $p(-273 /+61)-L u c$. Site-directed mutagenesis for the ER $\beta$ binding site construct was performed by overlapping extension PCR as previously described (2) and termed p(- $\Delta 734 /+61)$-Luc.

Luciferase assay. The primary hippocampal cells were plated in 6-well tissue culture dishes at $9 \times 10^{4}$ cells/well and used for transient transfection at day 7 in vitro (DIV). Cells were cotransfected with $1.5 \mu \mathrm{g}$ of luciferase reporter plasmid and $0.5 \mu \mathrm{g}$ of $\beta$-gaglactosidase expression vector $\mathrm{pCMV} \beta$ as an internal control. At $36 \mathrm{~h}$ after transfection, the cells were lysed to measure the luciferase activity using the Luciferase Assay System (Promega, Madison, WI, USA).

To study the regulation of ER $\beta$ on Tnfaip1 using the luciferase assay, the coding sequences of mouse $\operatorname{ER} \beta$ were amplified from mouse hippocampal cDNA and cloned into the eukaryotic expression vector pCMV-Myc. The construct was designated as pCMV-Myc-ER $\beta$. The luciferase reporter plasmid including the site-directed mutagenesis for the ER $\beta$ binding site and pCMV-Myc-ER $\beta$ were cotransfected.

ChIP. To detect the association of ER $\beta$ with Tnfaip1, chromatin immunoprecipitation (ChIP) was performed using overexpressed ER $\beta$ protein. The primary hippocampal cells were cultured and transfected with an ER $\beta$-expressing pCMV-Myc vector as previously described (23). ChIP was conducted following the recommended protocols (Active Motif). Specifically, enzymatic shearing was conducted for 10 min and IP was performed with the ER $\beta$ rabbit polyclonal

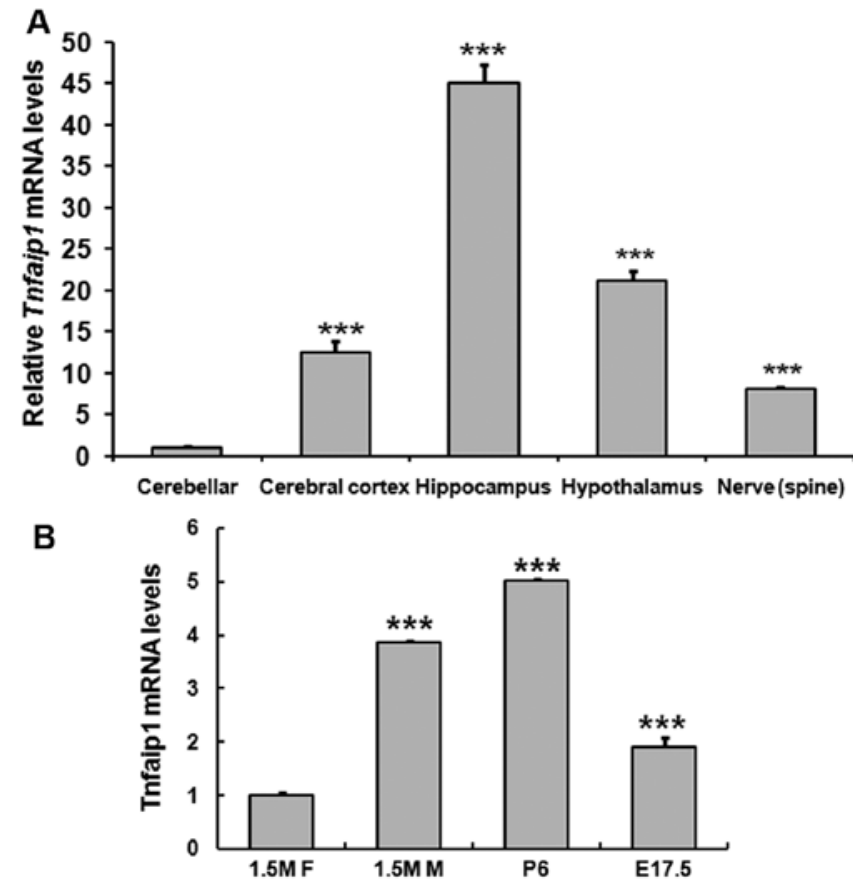

Figure 1. Tumor necrosis factor-induced protein 1 (Tnfaip1) mRNA levels in the mouse nerve system. Tissues were dissected and quantitative polymerase chain reaction (qRT-PCR) was performed. (A) Tnfaipl mRNA levels in the nerve system including cerebellar, cerebral cortex, hippocampus, hypothalamus and peripheral nerve, respectively. ${ }^{* * *} \mathrm{P}<0.001$ when cerebellar was compared with the cerebral cortex, hippocampus, hypothalamus and peripheral nerve. (B) Tnfaip1 mRNA levels in hippocampus of different age and gender. E17.5, embryonic 17.5 day; P6, postnatal 6 day; $1.5 \mathrm{M} \mathrm{F}$, 1.5-month-old female; $1.5 \mathrm{M} \mathrm{M}, 1.5$-month-old male. ${ }^{* * *} \mathrm{P}<0.001$ when 1.5 M F was compared with 1.5 M M, P6 or E17.5.

IgG (Sigma-Aldrich) at a concentration of $3 \mu \mathrm{g} / 100 \mu \mathrm{l}$ or the pre-immune mouse IgG as the negative control. Potential ER $\beta$ binding sites were predicted using the JASPAR TF Search program. PCR primers were designed flanking putative ER $\beta$ binding sites (forward, 5'-tcatgtggaaacagaaggctg-3' and reverse primer, 5'-agttcttcetgtgtctacgcc-3') and ChIP pull-down products were amplified for 30 cycles with a $60^{\circ} \mathrm{C}$ annealing temperature and imaged by agarose gel electrophoresis.

\section{Results}

Expression of Tnfaipl in nerve tissues. To study the function of Tnfaip1 in the nerve system, we compared the expression of Tnfaipl in nerve system using quantitative PCR (qPCR). The 1.5 M mouse tissues including cerebral cortex, hippocampus, cerebellar, hypothalamus, and nerve in spine were dissected and qPCR was performed as described in Materials and methods. Positive signals were detected in all the tissues. In these tissues, the a significant difference in Tnfaip1 expression in hippocampus and cerebellar with a 45-fold difference was identified (Fig. 1A). This result suggested that Tnfaip1 may play a critical role in the nerve system, particularly in the hippocampus.

Age- and gender-related difference in the expression of Tnfaipl in hippocampus. Previously it was reported that Tnfaip11 was involved in response to $\beta$-amyloid peptide accumulation in a transgenic $C$. elegans AD model (3). However, 


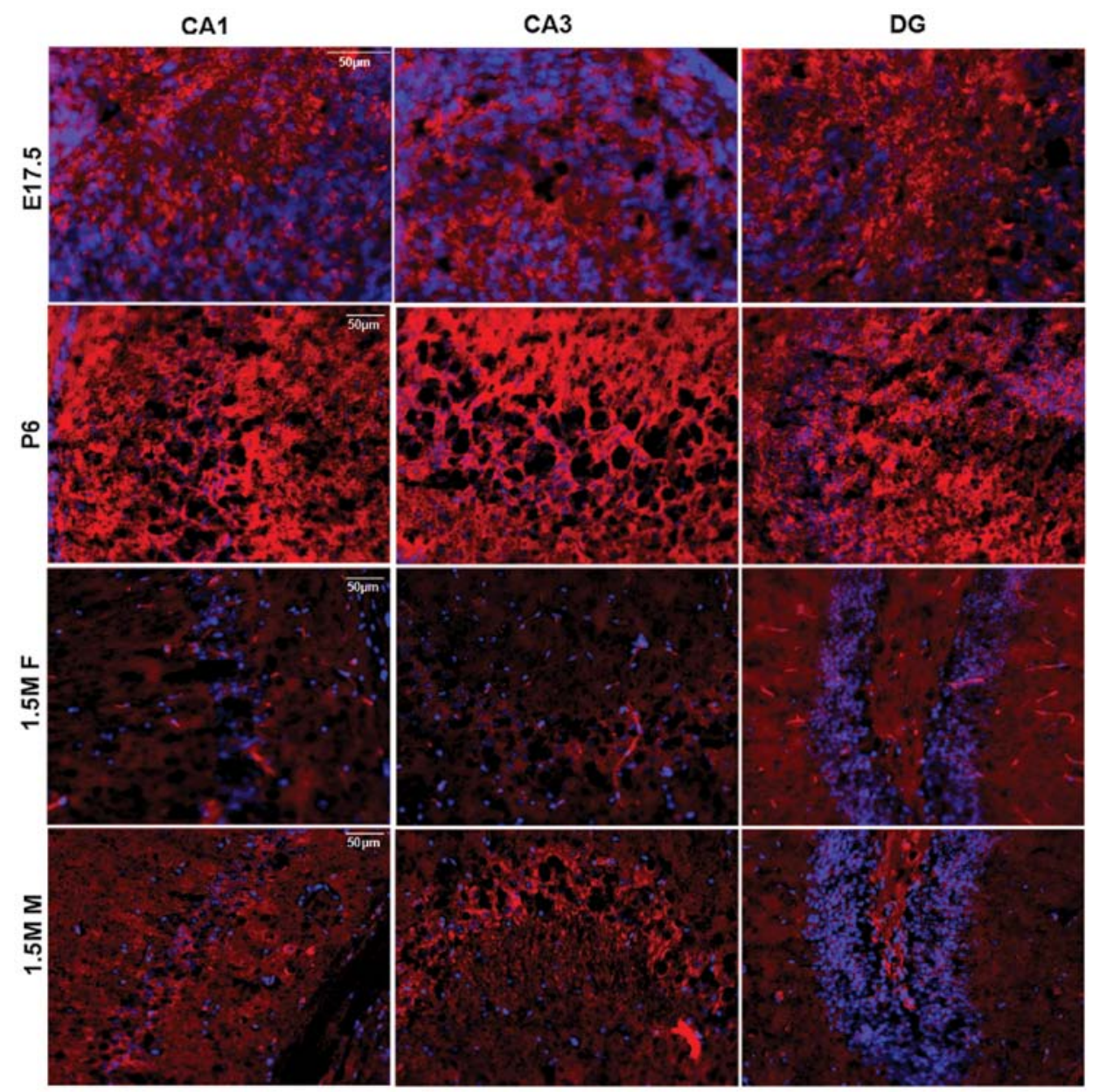

Figure 2. Fluorescent immunostaining in mouse hippocampus. The hippocampus of different age and gender was dissected, fixed, immunostained with anti-tumor necrosis factor induced protein 1 (Tnfaip1) antibody as the primary antibody (red) and DAPI for nucleus (blue). Blue is E17.5, embryonic 17.5 day; P6, postnatal 6 day; 1.5 M F, 1.5-month-old female; $1.5 \mathrm{M} \mathrm{M}$, 1.5-month-old male. CA1, CA3, DG were the subregions of hippocampus.

little is known with regard to the exact role of Tnfaipl in these diseases. Evidence that the parahippocampal cortex (including the entorhinal cortex) undergoes pathological changes during the early stages of the disease is theoretically significant (24). To investigate the role of Tnfaip1 in these diseases, three check points were selected: embryonic (E17.5), postnatal (P6), and adult $(1.5 \mathrm{M})$, with a focus on the expression of Tnfaip1 in hippocampus. The hippocampal tissues were dissected from mice of different age and gender. The results of qPCR showed that Tnfaip1 expression was low in E17.5, high in P6, and lowest in $1.5 \mathrm{M}$. Of note, at the $1.5 \mathrm{M}$ stage, male mice had a stronger expression than the female mice (Fig. 1B).

To map Tnfaip1 in hippocampus, the brains including the hippocampus were dissected, the slides were cut, and fluorescent immunostaining was performed using anti-Tnfaip1 antibodies as primary antibodies. Tnfaipl showed strong staining in the hippocampus of P6 mouse, but faint staining in the $1.5 \mathrm{M}$ female hippocampus. The $1.5 \mathrm{M}$ male exhibited stronger immunoreactivity than the female counterpart (Fig. 2). The results were consistent with qPCR results, showing age- and gender-related differences in the expression of Tnfaip1. The CA3 region can be divided into CA3a,b,c subareas (25). Fig. 2 shows data of only CA3a subareas, with the data of CA3b and $\mathrm{c}$ not being shown. Among the slides including all the ages and both genders, the pyramidal cells in CA3 regions including the CA3a,b,c subareas in the adult mouse have the strongest staining, higher than in the subregions of CA1 and DG (Fig. 2). These data suggested that Tnfaipl plays an important role in short-term memory and pattern separation of the geometry of environment through the CA3 region.

Effect of peripheral estrogen on the expression of Tnfaipl in hippocampus. Findings of previous studies suggest that the systemic estradiol treatment of ovariectomized animals caused an increase in the number of dendritic spines in this particular hippocampal region (18) and spine synapse density varied in response to fluctuating estrogen levels during the estrous cycle in female rats (19). To study the effect of systemic estrogen on the expression of Tnfaipl in hippocampus, the OVX mice were implanted with placebo pellet or $17 \beta$-estradiol pellet for 4 weeks. The entire brain tissue was dissected for fluorescent immunostaining and the hippocampus was dissected for western blot or real-time analysis. The Sham mice were used as the control. The results with fluorescent immunostaining showed that Tnfaip1 had stronger staining in the hippocampal CA3 subregion of OVX + placebo mice than the OVX + E2 or Sham mice (Fig. 3A-C). The staining was not different in the CA1 or DG subregions (data not shown). Furthermore, the mRNA level (Fig. 3D) and protein level (Fig. 3E) of Tnfaip1 in hippocampus was higher in the OVX + placebo mice than 

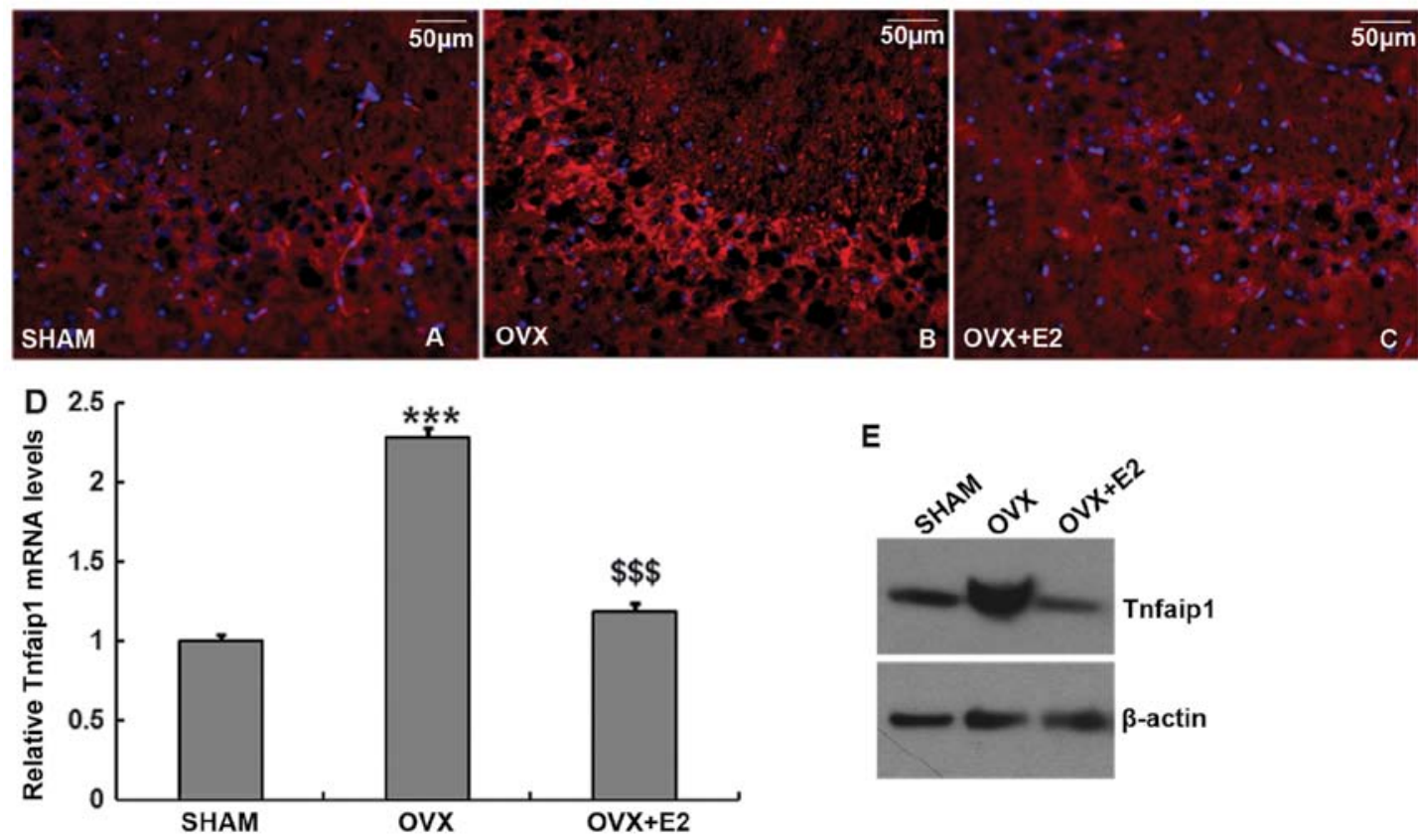

Figure 3. Effect of systemic estrogen on tumor necrosis factor-induced protein 1 (Tnfaip1) expression in mouse hippocampus. The ovariectomy (OVX) mice were implanted with a placebo pellet or 17ß-estradiol pellet for 4 weeks. (A-C) The entire brain tissue was dissected, fixed with $4 \%$ PFA and then immunostained with anti-Tnfaip1 antibody as primary antibody. The images show the signals in the CA3 subregions. (D) Quantitative polymerase chain reaction (qPCR). ${ }^{* * *} \mathrm{P}<0.001$ when the Sham group was compared with the OVX or OVX $+\mathrm{E} 2$ group. ${ }^{\$ \$ \$} \mathrm{P}<0.001$ when the OVX group was compared with the OVX $+\mathrm{E} 2$ group. (E) Western blot showing the protein level of Tnfaip1 in the hippocampus of Sham-operated mice, or mice treated with OVX or OVX + placebo.
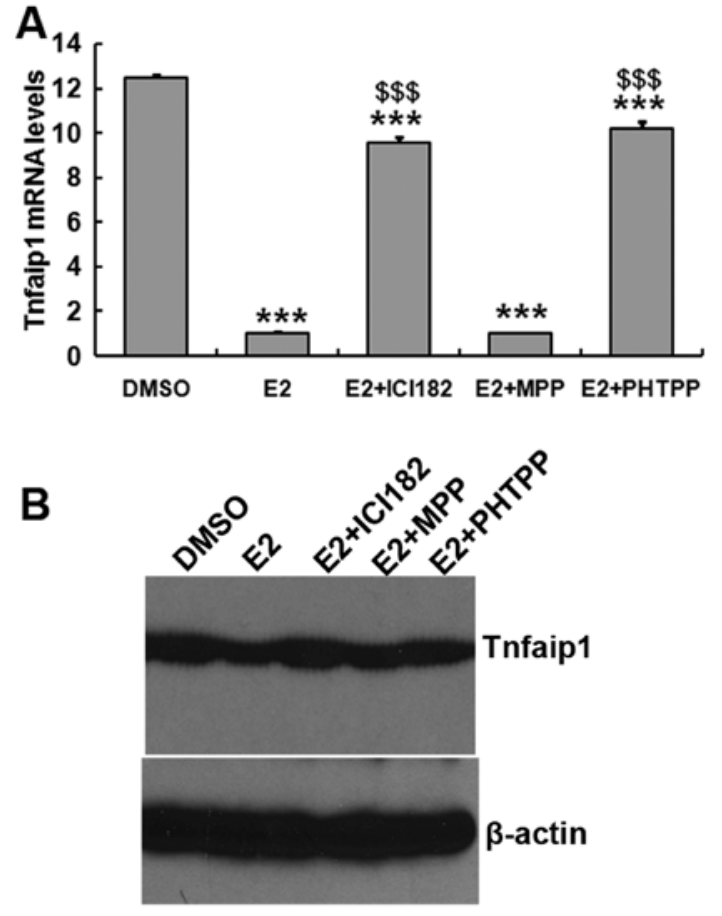

Figure 4. The inhibitory effect of estrogen on tumor necrosis factor-induced protein 1 (Tnfaip1) expression is reversed by certain estrogen antagonists in primary hippocampal neuronal cells. The cells were treated with either $1 \mu \mathrm{M}$ ER antagonist ICI182,780, or $1 \mu \mathrm{M}$ selective ER $\alpha$ antagonist MPP, or $5 \mu \mathrm{M}$ selective ER $\beta$ antagonist PHTPP, with the presence of $10 \mathrm{nM}$ $17 \beta$-estradiol (E2). Dimethylsulfoxide (DMSO) was used as a control. The results of (A) quantitative polymerase chain reaction (qPCR) and (B) western blotting are shown. ${ }^{* * *} \mathrm{P}<0.001$ when control using DMSO is compared with E2, E2 + ICI182,780, E2 + MPP and E2 + PHTPP. ${ }^{\$ \$ \$} \mathrm{P}<0.001$ when cells treated with E2 are compared with E2 + ICI182,780, E2 + MPP and E2 + PHTPP. in the OVX $+\mathrm{E} 2(\mathrm{P}<0.001)$ or in Sham mice $(\mathrm{P}<0.001)$. These results suggested that systemic estrogen inhibits Tnfaip1 expression in hippocampus.

Effect of estrogen on Tnfaipl expression in in vitro hippocampal neuronal cells. To examine the effect of estrogen on Tnfaip1 expression, hippocampal neuronal cells were prepared from mouse embryos and cultured in the presence or absence of E2. Tnfaip1 expression was lower in the cells treated with estrogen compared to the control (Fig. 4).

The classic estrogen receptors, known as $\mathrm{ER} \alpha$ and $\mathrm{ER} \beta$, are nuclear transcription factors that activate or repress gene expression (12). To determine whether ER $\alpha$ and ER $\beta$ were involved in the inhibition progress, the cells were treated with DMSO (control) or estrogen receptor antagonists (ICI182,780, MPP or PHTPP) in the presence of E2. Inhibition of Tnfaip1 expression by E2 was reversed by ER non-selective antagonist ICI182,780 and ER $\beta$-selective antagonist PHTPP, but not by ER $\alpha$-selective antagonist MPP. These data suggested that estrogen downregulates Tnfaipl expression in in vitro hippocampal cells, which was consistent with the in vivo results. Additionally, this function of estrogen may be mediated by ER $\beta$.

ER $\beta$ upregulates Tnfaipl expression by binding to the promoter of Tnfaipl. To examine the regulation of ER $\beta$ on Tnfaip1 expression, a promoter analysis was performed. The promoter region of mouse Tnfaip1 was predicted to be a 2166-bp genomic region. A series of 5'-deletion promoter regions upstream of the first exon of the Tnfaipl gene were amplified and inserted into the promoterless luciferase reporter construct. Insertion of longer regions $(-2166 /+61,-1072 /+61$ and $-734 /+61)$ significantly enhanced the promoter activity 


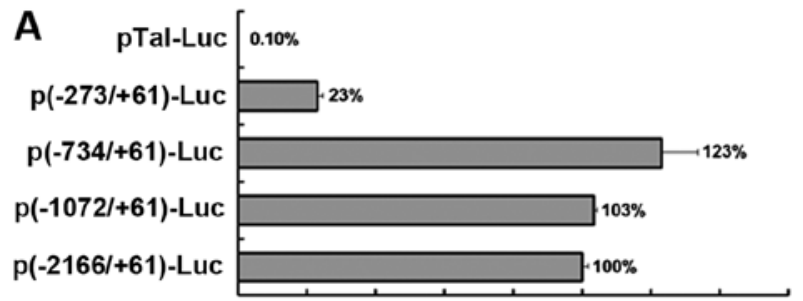

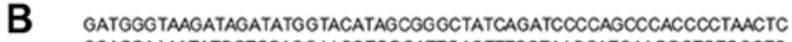
CCACCAAAATATGCTOCAGGAACGTGGGATTGAGTTTCCTAAGCATGAAGGCTGTGCCTG GCTCTACGCTCCCTGGCTCTCCCAGGTOCGCAGGCACAACAAATAGTTGTAGCTGTGGAG TITGGAGAAGGCGTAGACACAGGAAGAACTGGGCAAATGGCGCGGCTGTCTITACTAAAT SP1

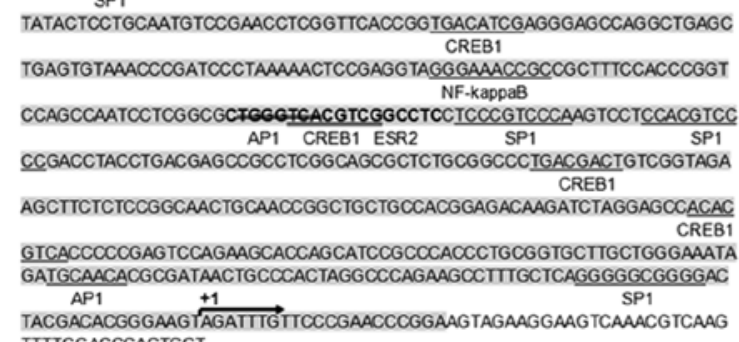

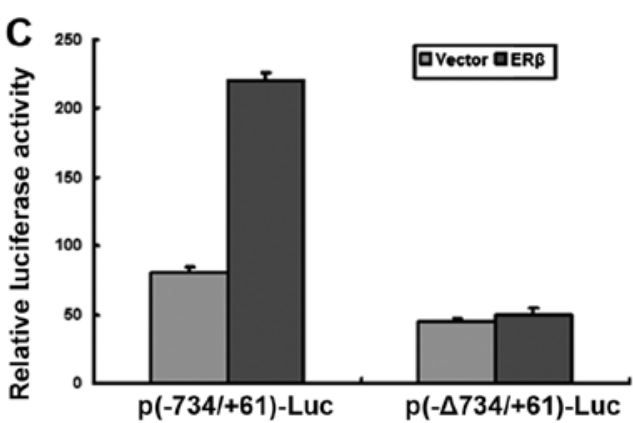

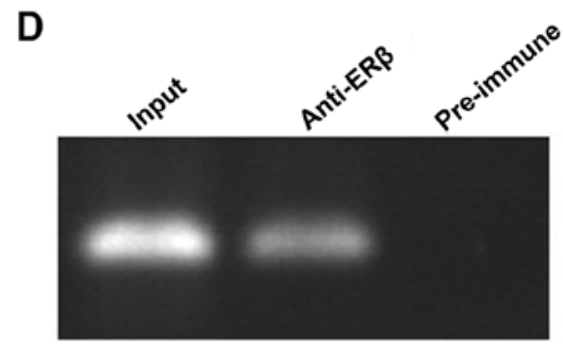

Figure 5. Promoter analysis of tumor necrosis factor-induced protein 1 (Tnfaip1). (A) The plasmids, containing sequentially deleted fragments of the mouse Tnfaip1 5'-flanking region, were transfected into hippocampal neuronal cells. Luciferase activities were measured at $36 \mathrm{~h}$ post-transfection. The luciferase activity values of the other regions were relative to that of the $-2166 /+61$ region, which was set as $100 \%$. (B) Sequence analysis for the 5'-flanking region $(-734 /+61)$ of mouse Tnfaipl gene. The transcription factor binding sites were predicted using the online software JASPAR and labeled with an underline marker. The $\mathrm{CpG}$ island was scanned and labeled using the shade marker. (C) The plasmid, containing the $-734 /+61$ or mutated $\Delta 734 /+61$, was co-transfected with pCMV-Myc-ER $\beta$ or control vector pCMV-Myc into cells and then the luciferase activity was measured at $36 \mathrm{~h}$ post-transfection. (D) Immunoprecipitation (ChIP) assay for interaction between ER $\beta$ and the promoter region of mouse Tnfaip1 gene. Formaldehyde cross-linked chromatin from the cells transfected with the ER $\beta$ expression vector was subjected to ChIP experiments. Immunoprecipitations were performed using anti-ER $\beta$ antibody. The pre-immune mouse IgG was used as the negative control. After isolation of bound DNA, polymerase chain reaction (PCR) was performed on a 409-bp fragment including the ER $\beta$ potential binding site in Tnfaip1 promoter. Input, PCR performed on DNA without immunoprecipitation.

compared to the promoterless luciferase control (Fig. 5A). The deletion $-734 /+61$ had the strongest enhancement, followed by the $-1072 /+61$ deletion. Both were stronger than the longest insertion $(-2166 /+61)$. However, the deletion $-237 /+61$ showed a significant decrease in promoter activity. These results showed that the upstream region $-2166 /+61$ of the mouse Tnfaipl gene contained a functional promoter and the main regulatory element resided in the range of -734 to +61 .

To further determine the potential estrogen response element (ERE), the region -734 to +61 was scanned using the JASPAR and CpG-prediction software. No classical TATA box or CCAAT box was found in the promoter region. Instead, a $\mathrm{CpG}$ island was detected from -607 to +21 , whose sequence was shaded in Fig. 5B. A potential ER $\beta$ (known as ERS2 in the software) binding site was identified in this region. However, the ER $\alpha$ binding site was not found (Fig. 5B). To study the regulation of ER $\beta$ on Tnfaip1 expression, the $\mathrm{p}(-734 /+61)$ Luc or the mutant $p(-\Delta 734 /+61)$-Luc was co-transfected with pCMV-Myc-ER $\beta$ into cultured cells. The luciferase activity analysis revealed that the overexpression of $\operatorname{ER} \beta$ enhanced the Tnfaip1 promoter activity, compared to the empty or mutated vector (Fig. 5C). To confirm the involvement of ER $\beta$ in the Tnfaip1 promoter regulation, we performed ChIP assay using the hippocampal primary cells. (Fig. 5D) The cross-linked mouse Tnfaip1 promoter-ER $\beta$ complexes immunoprecipitated with anti-ER $\beta$ antibody were detected by PCR amplification with primers covering the potential binding sites of mouse Tnfaip1 promoter. No band was detected with a non-specific antibody as a negative control. These results demonstrated that ER $\beta$ can directly bind to the Tnfaip1 promoter, providing novel evidence for Tnfaip1 transcriptional regulation.

\section{Discussion}

Tnfaip1 was initially identified as a tumor necrosis factor $\alpha$-induced protein (1). Although TNF- $\alpha$ treatment was reported to protect hippocampal neurons against $A \beta$ toxicity (26), no study has shown Tnfaip1 to be associated with $A \beta$ toxicity since its identification. Until 10 years ago, the homolog gene of Tnfiap1 was upregulated in response to A $\beta$ treatment, using the $C$. elegans AD model. The hippocampus is a complex structure, most commonly known for its role in learning and memory. The hippocampus consists of CA1, CA3, and the dentate gyrus regions, and comprises distinct neuronal cell types including pyramidal neurons, interneurons, and granule cells. The hippocampus has been the target for extensive studies regarding spatial and nonspatial memory, long-term potentiation, adult neurogenesis, neurodegeneration and neurodegenerative diseases, such as Alzheimer's. In this study, we focused on the expression of Tnfaipl in the mouse hippocampus, which is one of the brain regions affected early in the AD process. The present study showed high mRNA levels in hippocampus (Fig. 1A), and strong staining in the CA3 subregions in mice of all ages and both genders (Fig. 2). The data suggest that Tnfaip1 potentially plays an important role in the hippocampus. However, the exact function remains to be investigated in Tnfaip1 knock-out or transgenic mice. 
Despite being markedly different in anatomy, the subregions CA3, CA1 and dentate gyrus (DG) in hippocampus have distinct functions $(27,28)$. In particular, the extensive excitatory recurrent connections of region $\mathrm{CA} 3$ have been suggested to play an important role in the encoding and retrieval of new spatial information within short-term memory $(29,30)$ and spatial information requiring multiple trials $(31,32)$. The CA3 region also supports sequential processing of information in cooperation with CA1 (32) and processing of the geometry of the environment in cooperation with DG $(33,34)$. In this study, our data showed that the expression of Tnfaipl was stronger in the CA3 region of the adult hippocampus (Fig. 2). The high expression of Tnfaipl suggests its important role in the above-mentioned functions. A recent study has highlighted functional deficits in the DG/CA3 hippocampal subfields in animal models of AD pathology and in patients in the early stages of dementia (35). Additionally, its findings showed that activity in the DG/CA3 networks was compromised in transgenic mice expressing an APP (amyloid precursor protein) mutation (35). To the best of our knowledge, the present study provides new evidence for the association between Tnfaip1 and $\mathrm{AD}$ although it was previously reported that the homolog gene of Tnfaipl was upregulated in the superior frontal gyrus and cerebellum but not significantly increased in the hippocampus of a transgenic C. elegans AD model (3). This conflict may have resulted from the difference in study species, analytical methods and models.

In this study, the age- and gender-related expression of Tnfaip1 in hippocampus was detected using qPCR (Fig. 1B) and fluorescent immunostaining (Fig. 2). The result is consistent with the study of Wolf et al (1), who investigated the developmental expression in heart and liver. Our data provide complementary evidence withe regard to the developmental expression of Tnfaip1, particularly in the hippocampus. Based on the result of the age- and gender-related expression of Tnfaip1, we hypothesized that Tnfaip1 is regulated by estrogen. Previous findings strongly suggest that hippocampal neurons are able to synthesize estrogens de novo $(14,15)$, and hippocampus-derived endogenous estrogen is essential for hippocampal synaptic plasticity (16). However, a number of other studies have suggested that peripheral estrogen exerts a protective effect in hippocampal neurons. Woolley et al (19) were the first to show cyclic fluctuations in dendritic spine density as estradiol and progesterone levels vary across the estrous cycle in adult female rats. At the same time, they also reported that OVX resulted in a decrease in dendritic spine density in the hippocampus in female rat, which could be prevented by systemic estradiol treatment. Several subsequent experiments confirmed the connection between ovarian estrogens and spine synapse density in rodents (36) and in non-human primates (37). Another study found that synaptic plasticity highly depends on endogenous estrogen and cannot be influenced by exogenous estrogen (16). A study performed by Leranth et al (38) may help to explain this discrepancy. Their studies showed an indirect action of estrogen on hippocampal spinogenesis, i.e., the systemic application of estradiol results in an increase of spine density indirectly via the subcortical regions. Similar results strongly suggest that systemic estrogen effects on hippocampus may be mediated by various subcortical regions, such as medial septum/diagonal band of
Broca (MSDB), the supramammillary area (SUM) and the median raphe (MR) (39-41). The MSDB may play a key role in this process since it is not only sensitive to local estrogen stimulation but its septohippocampal neurons receive stimulatory signals from the SUM and MR $(42,43)$. To examine the regulation of systemic estrogen on Tnfaipl expression in hippocampus, OVX and Sham mice were used. Compared to the control, Sham mice, a high expression was detected in the hippocampus of OVX mice using fluorescent immunostaining, qPCR and western blot analysis (Fig. 3). The enhanced expression was reversed by complementary estrogen (Fig. 3). These data provide novel evidence that systemic estrogen downregulates Tnfaip1 expression in hippocampus.

The classic estrogen receptors, known as ER $\alpha$ and ER $\beta$, are nuclear transcription factors that activate or repress gene expression (12). Hippocampal neurons in tissue sections as well as in slice cultures and dispersion cultures express ER $\alpha$ and ER $\beta$ mRNA $(13,14)$. To determine whether ER $\alpha$ and ER $\beta$ were involved in the inhibition process, the primary hippocampal cells were cultured and treated with estrogen receptor antagonists. In cultured hippocampal neuronal cells, estrogen inhibited Tnfaip1 expression compared to the control (Fig. 4). The decrease of Tnfaip1 expression was reversed by both the ER non-selective antagonist and ER $\beta$-selective antagonist, but not by the ER $\alpha$-selective antagonist (Fig. 4). These data suggest that estrogen actually downregulated Tnfaip1 expression in vitro, consistent with the in vivo results (Fig. 3). These results also suggest that the regulation of estrogen on Tnfaip1 expression may be through ER $\beta$ mediation. Previous studies have described the acute, membrane-initiated signaling actions of E2 in the brain through multiple signaling pathways (44), such as G-protein-activated pathways (45), the mitogen-activated protein kinase (MAPK) pathway $(46,47)$ and the phosphatidylinositol 3-kinase (PI3K)/Akt pathway (48-50). At least some of these rapid actions of E2 cannot be attributed to classical nuclear-initiated steroid signaling of ER $\alpha$ or ER $\beta$. There are several potential candidates for novel membrane ERs including ER-X and two G-protein-coupled receptors, GPR30 and Gq-mER $(51,52)$. However, the mechanism whereby membrane ERs activate the neuroprotective cascade is largely unknown.

Mounting evidence suggests that $\operatorname{ER} \alpha$ and $\operatorname{ER} \beta$ can regulate transcription of some of these 'estrogen-responsive' genes by interacting with other DNA-bound transcription factors, such as specificity protein-1 (SP-1) and activator protein-1 (AP-1), rather than binding directly to DNA $(53,54)$. When scanning the promoter region of the mouse Tnfaip1 gene, we found the potential binding sites of ER $\beta$ and other transcription factors (Fig. 5B), but no binding sites of ER $\alpha$. This may explain why the effect of estrogen on Tnfaipl expression occurs through the ER $\beta$ but not the ER $\alpha$ pathway. ER $\beta$ upregulates Tnfaip1 expression (Fig. 5C) and is able to physically bind to the Tnfaip1 promoter (Fig. 5D). In the study of Prange-Kiel et al (14), treating the primary hippocampal neurons with additional estradiol resulted in the upregulation of $E R \alpha$ and downregulation of $E R \beta$, as immunohistochemistry and subsequent semiquantitative image analysis revealed. Expression of ERs is known to be regulated by estrogen in both reproductive system $(55,56)$ and brain regions $(57)$. The possible mechanism can be deduced based on these studies 
and our study for the effect of estrogen on Tnfaip1 expression. Estrogen upregulates ER $\alpha$ and subsequently downregulates ER $\beta$. Although ER $\beta$ is able to upregulate Tnfaip1 expression, its function is inhibited by estrogen. To the best of our knowledge, our data provide a novel regulatory mechanism Tnfaip1 expression in hippocampus. This study also elucidated the potential function of Tnfaip1 in hippocampus-related disease, such as AD, which may be affected by the estrogen level.

\section{Acknowledgements}

This study was supported by the foundation from the National Natural Science Foundation of China (81173177), the Science and Technology Planning Project of Hunan (2009FJ3012), the Educational Commission of Hunan (09C1062 and 10C0018), the Environmental Scientific Research Foundation of Hunan (2009364), China.

\section{References}

1. Wolf FW, Marks RM, Sarma V, et al: Characterization of a novel tumor necrosis factor-alpha-induced endothelial primary response gene. J Biol Chem 267: 1317-1326, 1992.

2. Zhou J, Hu X, Xiong X, et al: Cloning of two rat PDIP1 related genes and their interactions with proliferating cell nuclear antigen. J Exp Zool A Comp Exp Biol 303: 227-240, 2005.

3. Link CD, Taft A, Kapulkin V, et al: Gene expression analysis in a transgenic Caenorhabditis elegans Alzheimer's disease model Neurobiol Aging 24: 397-413, 2003.

4. Zhou J, Ren K, Liu X, Xiong X, Hu X and Zhang J: A novel PDIP1-related protein, KCTD10, that interacts with proliferating cell nuclear antigen and DNA polymerase delta. Biochim Biophys Acta 1729: 200-203, 2005.

5. Smith TC, Fang Z and Luna EJ: Novel interactors and a role for supervillin in early cytokinesis. Cytoskeleton (Hoboken) 67: 346-364, 2010.

6. Liu XW, Lu FG, Zhang GS, et al: Proteomics to display tissue repair opposing injury response to LPS-induced liver injury. World J Gastroenterol 10: 2701-2705, 2004.

7. Yang LP, Zhou AD, Li H, et al: Expression profile in the cell lines of human TNFAIP1 gene. Yi Chuan 28: 918-922, 2006 (In Chinese).

8. Yang L, Liu N, Hu X, et al: CK2 phosphorylates TNFAIP1 to affect its subcellular localization and interaction with PCNA. Mol Biol Rep 37: 2967-2973, 2010

9. Gupta J, Gaikwad AB and Tikoo K: Hepatic expression profiling shows involvement of PKC epsilon, DGK eta, Tnfaip, and Rho kinase in type 2 diabetic nephropathy rats. J Cell Biochem 111: 944-954, 2010

10. Kuiper GG, Shughrue PJ, Merchenthaler I and Gustafsson JA: The estrogen receptor beta subtype: a novel mediator of estrogen action in neuroendocrine systems. Front Neuroendocrinol 19: 253-286, 1998.

11. Hultcrantz M, Simonoska $R$ and Stenberg AE: Estrogen and hearing: a summary of recent investigations. Acta Otolaryngol 126: 10-14, 2006.

12. Nilsson S, Mäkelä S, Treuter E, et al: Mechanisms of estrogen action. Physiol Rev 81: 1535-1565, 2001.

13. Wehrenberg U, Prange-Kiel J and Rune GM: Steroidogenic factor-1 expression in marmoset and rat hippocampus: co-localization with StAR and aromatase. J Neurochem 76: 1879-1886, 2001.

14. Prange-Kiel J, Wehrenberg U, Jarry H and Rune GM: Para/autocrine regulation of estrogen receptors in hippocampal neurons. Hippocampus 13: 226-234, 2003.

15. Kretz O, Fester L, Wehrenberg U, et al: Hippocampal synapses depend on hippocampal estrogen synthesis. J Neurosci 24 5913-5921, 2004.

16. Prange-Kiel J and Rune GM: Direct and indirect effects of estrogen on rat hippocampus. Neuroscience 138: 765-772, 2006.

17. Green PS, Yang SH, Nilsson KR, Kumar AS, Covey DF and Simpkins JW: The nonfeminizing enantiomer of 17beta-estradiol exerts protective effects in neuronal cultures and a rat model of cerebral ischemia. Endocrinology 142: 400-406, 2001.
18. Gould E, Woolley CS, Frankfurt M and McEwen BS: Gonadal steroids regulate dendritic spine density in hippocampal pyramidal cells in adulthood. J Neurosci 10: 1286-1291, 1990.

19. Woolley CS, Gould E, Frankfurt M and McEwen BS: Naturally occurring fluctuation in dendritic spine density on adult hippocampal pyramidal neurons. J Neurosci 10: 4035-4039, 1990.

20. Moran AL, Warren GL and Lowe DA: Removal of ovarian hormones from mature mice detrimentally affects muscle contractile function and myosin structural distribution. J Appl Physiol 100: 548-559, 2006.

21. Moran AL, Nelson SA, Landisch RM, Warren GL and Lowe DA: Estradiol replacement reverses ovariectomy-induced muscle contractile and myosin dysfunction in mature female mice. $\mathrm{J}$ Appl Physiol 102: 1387-1393, 2007.

22. Flavin MP, Coughlin K and Ho LT: Soluble macrophage factors trigger apoptosis in cultured hippocampal neurons. Neuroscience 80: 437-448, 1997.

23. Viesselmann C, Ballweg J, Lumbard D and Dent EW: Nucleofection and primary culture of embryonic mouse hippocampal and cortical neurons. J Vis Exp: pii: 2373. doi: 10.3791/2373, 2011.

24. Hyman BT, Van Hoesen GW, Kromer LJ and Damasio AR: Perforant pathway changes and the memory impairment of Alzheimer's disease. Ann Neurol 20: 472-481, 1986.

25. Li XG, Somogyi P, Ylinen A and Buzsaki G: The hippocampal CA3 network: an in vivo intracellular labeling study. J Comp Neurol 339: 181-208, 1994.

26. Barger SW, Hörster D, Furukawa K, Goodman Y, Krieglstein J and Mattson MP: Tumor necrosis factors alpha and beta protect neurons against amyloid beta-peptide toxicity: evidence for involvement of a kappa B-binding factor and attenuation of peroxide and $\mathrm{Ca}^{2+}$ accumulation. Proc Natl Acad Sci USA 92: 9328-9332, 1995.

27. Amaral DG and Witter MP: The three-dimensional organization of the hippocampal formation: a review of anatomical data. Neuroscience 31: 571-591, 1989.

28. Hasselmo ME: The role of hippocampal regions CA3 and CA1 in matching entorhinal input with retrieval of associations between objects and context: theoretical comment on Lee et al (2005). Behav Neurosci 119: 342-345, 2005.

29. Lee I and Kesner RP: Differential contribution of NMDA receptors in hippocampal subregions to spatial working memory. Nat Neurosci 5: 162-168, 2002.

30. Kesner RP, Lee I and Gilbert P: A behavioral assessment of hippocampal function based on a subregional analysis. Rev Neurosci 15: 333-351, 2004.

31. Hunsaker MR, Allan KD and Kesner RP: Role of dCA3 efferents via the fimbria in the acquisition of a delay nonmatch to place task. Hippocampus 17: 494-502, 2007.

32. Lee I, Jerman TS and Kesner RP: Disruption of delayed memory for a sequence of spatial locations following CA1- or CA3-lesions of the dorsal hippocampus. Neurobiol Learn Mem 84: 138-147, 2005.

33. Tanila H: Hippocampal place cells can develop distinct representations of two visually identical environments. Hippocampus 9: 235-246, 1999.

34. Leutgeb JK, Leutgeb S, Moser MB and Moser EI: Pattern separation in the dentate gyrus and CA3 of the hippocampus. Science315: 961-966, 2007.

35. Palmer A and Good M: Hippocampal synaptic activity, pattern separation and episodic-like memory: implications for mouse models of Alzheimer's disease pathology. Biochem Soc Trans 39: 902-909, 2011.

36. McEwen B: Estrogen actions throughout the brain. Recent Prog Horm Res 57: 357-384, 2002.

37. Leranth C, Shanabrough M and Redmond DE Jr: Gonadal hormones are responsible for maintaining the integrity of spine synapses in the CA1 hippocampal subfield of female nonhuman primates. J Comp Neurol 447: 34-42, 2002.

38. Leranth C, Shanabrough M and Horvath TL: Hormonal regulation of hippocampal spine synapse density involves subcortical mediation. Neuroscience 101: 349-356, 2000.

39. Prange-Kiel J, Rune GM and Leranth C: Median raphe mediates estrogenic effects to the hippocampus in female rats. Eur J Neurosci 19: 309-317, 2004.

40. Leranth $C$ and Shanabrough M: Supramammillary area mediates subcortical estrogenic action on hippocampal synaptic plasticity. Exp Neurol 167: 445-450, 2001. 
41. Lâm TT and Leranth C: Role of the medial septum diagonal band of Broca cholinergic neurons in oestrogen-induced spine synapse formation on hippocampal CA1 pyramidal cells of female rats. Eur J Neurosci 17: 1997-2005, 2003.

42. Leranth C, Shanabrough M and Horvath TL: Estrogen receptoralpha in the raphe serotonergic and supramammillary area calretinin-containing neurons of the female rat. Exp Brain Res 128: 417-420, 1999.

43. Leranth C and Vertes RP: Median raphe serotonergic innervation of medial septum/diagonal band of broca (MSDB) parvalbumincontaining neurons: possible involvement of the MSDB in the desynchronization of the hippocampal EEG. J Comp Neurol 410: 586-598, 1999.

44. Bryant DN, Sheldahl LC, Marriott LK, Shapiro RA and Dorsa DM: Multiple pathways transmit neuroprotective effects of gonadal steroids. Endocrine 29: 199-207, 2006.

45. Kelly MJ and Rønnekleiv OK: Membrane-initiated estrogen signaling in hypothalamic neurons. Mol Cell Endocrinol 290: $14-23,2008$

46. Singer CA, Figueroa-Masot XA, Batchelor RH and Dorsa DM: The mitogen-activated protein kinase pathway mediates estrogen neuroprotection after glutamate toxicity in primary cortical neurons. J Neurosci 19: 2455-2463, 1999.

47. Mize AL, Shapiro RA and Dorsa DM:Estrogen receptor-mediated neuroprotection from oxidative stress requires activation of the mitogen-activated protein kinase pathway. Endocrinology 144: 306-312, 2003.

48. Honda K, Sawada H, Kihara T, et al: Phosphatidylinositol 3-kinase mediates neuroprotection by estrogen in cultured cortical neurons. J Neurosci Res 60: 321-327, 2000.
49. Harms C, Lautenschlager M, Bergk A, et al: Differential mechanisms of neuroprotection by 17 beta-estradiol in apoptotic versus necrotic neurodegeneration. J Neurosci 21: 2600-2609, 2001.

50. Cimarosti H, Zamin LL, Frozza R, et al: Estradiol protects against oxygen and glucose deprivation in rat hippocampal organotypic cultures and activates Akt and inactivates GSK-3beta. Neurochem Res 30: 191-199, 2005.

51. Funakoshi T, Yanai A, Shinoda K, Kawano MM and Mizukami Y: $\mathrm{G}$ protein-coupled receptor 30 is an estrogen receptor in the plasma membrane. Biochem Biophys Res Commun 346: 904-910, 2006

52. Toran-Allerand CD: Estrogen and the brain: beyond ER-alpha, ER-beta, and 17beta-estradiol. Ann NY Acad Sci 1052: 136-144, 2005.

53. Gruber CJ, Gruber DM, Gruber IM, Wieser F and Huber JC: Anatomy of the estrogen response element. Trends Endocrinol Metab 15: 73-78, 2004.

54. Paech K, Webb P, Kuiper GG, et al: Differential ligand activation of estrogen receptors ERalpha and ERbeta at AP1 sites. Science 277: 1508-1510, 1997.

55. Xiao CW and Goff AK: Hormonal regulation of oestrogen and progesterone receptors in cultured bovine endometrial cells. J Reprod Fertil 115: 101-109, 1999.

56. Murata T, Narita K, Honda K and Higuchi T: Changes of receptor mRNAs for oxytocin and estrogen during the estrous cycle in rat uterus. J Vet Med Sci 65: 707-712, 2003.

57. Tena-Sempere M, Navarro VM, Mayen A, Bellido C and Sanchez-Criado JE: Regulation of estrogen receptor (ER) isoform messenger RNA expression by different ER ligands in female rat pituitary. Biol Reprod 70: 671-678, 2004. 